\title{
Comparison of microleakage between bulk-fill flowable and nanofilled resin-based composites
}

\author{
EMAN I. ALSAGOB ${ }^{1,2, *}$, DAVID N. BARDWELL ${ }^{2}$, ALA O. ALI $^{2}$, SAMER G. KHAYAT $^{2}$, \\ PAUL C. STARK ${ }^{3}$
}

\author{
${ }^{1}$ Department of Preventive Dental Sciences, College of Dentistry, Princess Nourah bint Abdulrahman University, Riyadh, Saudi Arabia \\ ${ }^{2}$ Department of Prosthodontics and Operative Dentistry, School of Dental Medicine, Tufts University, Boston, MA, USA \\ ${ }^{3}$ Department of Advanced and Graduate Education, School of Dental Medicine, Tufts University, Boston, MA, USA \\ *Corresponding author: Eman I. AlSagob; Department of Preventive Dental Sciences, College of Dentistry, Princess Nourah bint Abdulrahman \\ University, Airport Road, Narjes Neighborhood, P.O. Box 84428, Riyadh 11671, Saudi Arabia; \\ Phone: +96 655517 8877; Fax: +96 611824 0874; E-mail: eialsagob@pnu.edu.sa
}

(Received: December 17, 2017; Revised manuscript received: January 15, 2018; Accepted: January 15, 2018)

\begin{abstract}
Aim: The objective was to compare the marginal leakage (silver nitrate uptake) of nanohybrid resin-based composite (RBC) and two bulkfill flowable RBCs with specific clinical protocols. Methods: Four experimental groups of RBC were investigated including conventional composite Filtek $^{\mathrm{TM}}$ Supreme in $2 \mathrm{~mm}$ increment (FS2), Filtek ${ }^{\mathrm{TM}}$ Supreme in $4 \mathrm{~mm}$ increment (FS4), Filtek ${ }^{\mathrm{TM}}$ Supreme Flowable (BFF), and SureFil ${ }^{\circledR}$ SDR ${ }^{\circledR}$ flow (SDR). Class II box preparation $(4 \times 4 \times 3 \mathrm{~mm})$ in extracted intact human molars was carried out and restored using the experimental groups, all according to the manufacturers' recommendations except FS4. Samples were aged by thermocycling $(2,000$ cycles $)$. Microleakage was calculated by measuring dye penetration in sectioned teeth using a stereomicroscope. Level of significance was set at $P<0.05$. Results: BFF and FS2 exhibited the least dye penetration and microleakage measurement with no significant difference between the two groups, followed by SDR. FS4 showed the highest microleakage with significant difference in comparison with BFF and FS2. Gingival microleakage was found to be significantly higher than occlusal microleakage. Conclusion: The microleakage of the bulk-fill composites BFF and SDR are comparable with conventional composite FS2; however, it is more predictable to use FS2.
\end{abstract}

Keywords: resin-based composite, bulk fill, flowable, microleakage, thermocycling

\section{Introduction}

Polymerization shrinkage remains a challenge, and one of the leading causes of secondary caries around resin-based composites (RBCs), which is the primary season for the clinical replacement of RBCs [1,2]. Research has focused on improving placement techniques, materials, and composite formulation, primarily the material's polymetric matrix, to develop systems with reduced polymerization shrinkage and polymerization shrinkage stress [3]. Although low-shrinking composite resins are desirable, noteworthy that there are a number of factors that determine shrinkage stress of a restoration. These include cavity geometry, the type of material, and application technique [4].
An incremental layering technique remains the gold standard to restore cavity preparation exceeding $2 \mathrm{~mm}$, due to several reasons besides minimizing gap formation and polymerization stress, such as achieving adequate bonding of composite to tooth tissue and ensuring complete polymerization of RBC [5-9]. The latest generations of flowable composites, i.e., bulk-fill flowable composites, have higher filler content and claim to have improved mechanical properties, making them preferred for larger posterior restorations [10]. Furthermore, the filling procedures are simplified and expedited by flowable composites that can be placed in bulk up to 4-mm thickness, without negatively affecting the polymerization shrinkage, cavity adaptation, or the degree of conversion [11-14].

This is an open-access article distributed under the terms of the Creative Commons Attribution-NonCommercial 4.0 International License, which permits unrestricted use, distribution, and reproduction in any medium for non-commercial purposes, provided the original author and source are credited, a link to the CC License is provided, and changes - if any - are indicated. 
Two recent studies have examined bulk-fill flowable RBCs' mechanical and physical properties. Czasch and Ilie [14] compared between two bulk-fill flowable RBCs, Venus Bulk Fill (Heraeus Kulzer), and SureFil SDR Flow, (DENTSPLY Caulk, Milford, DE, USA) and found that SDR had better mechanical properties despite a lower degree of conversion than Venus Bulk Fill. In addition, they found that a polymerization time of $20 \mathrm{~s}$ for $4-\mathrm{mm}$ bulk placed increments of either material seems to be sufficient. Another study conducted by Moorthy et al. [15] compared conventional RBC with two bulk-fill flowable RBCs, SDR (DENTSPLY Caulk) and X-tra base (VOCO GmbH, Cuxhaven, Germany), and found that the bulk-fill flowable composite groups had significantly less cuspal deflection than conventional RBC, with no difference in cervical microleakage among the groups. These two studies use a 4-mm filling technique, which goes against the convention of using incremental layering for cavity preparations exceeding $2 \mathrm{~mm}$. This contemporary bulk-fill flowable RBC and its new technique have few independent studies to validate it and need further evaluation [16]. Microleakage measurement provides an assessment of the marginal adaptation by evaluating dye penetration between the tested material and the tooth structure $[17,18]$. The longevity of a composite restoration is mainly affected by the microleakage; thus, it is essential to evaluate it $[19,20]$.

The aim of this study was to evaluate the marginal microleakage, through evaluating dye penetration, of the bulk-fill flowable RBCs in comparison with the conventional RBC (nanohybrid), utilizing incremental and bulk-filling technique. Two hypotheses were investigated: (a) the nanohybrid RBC Filtek ${ }^{\mathrm{TM}}$ Supreme Ultra Universal Restorative will exhibit less marginal leakage than two bulk-fill flowable RBCs, Filtek ${ }^{\mathrm{TM}}$ Bulk Fill Flowable Restorative and SureFil ${ }^{\circledR} \mathrm{SDR}^{\circledR}$ flow and (b) the nanohybrid $\mathrm{RBC}$ when restored in $2 \mathrm{~mm}$ increments will exhibit less marginal leakage than $4-\mathrm{mm}$ bulk.

\section{Materials and Methods}

\section{Study samples}

Twenty-four caries-free molar teeth with hypoplastic defects or cracks were used. The teeth were cleaned with an ultrasonic scaler (Cavitron GEN-119, SPS ${ }^{\text {TM }}$, DENTSPLY, PA, USA) to remove saliva, debris, and calculus deposits. They were placed in distilled water at room temperature to prevent them from drying. The teeth were embedded in autopolymerizing acrylic resin (Technovit, Heraeus Kulzer, Germany) using a plastic mounting template (Ultradent Product Inc., UT, USA) with the experimental surface of the tooth exposed (Fig. 1). The teeth were stored afterward in distilled water at room temperature at all times.

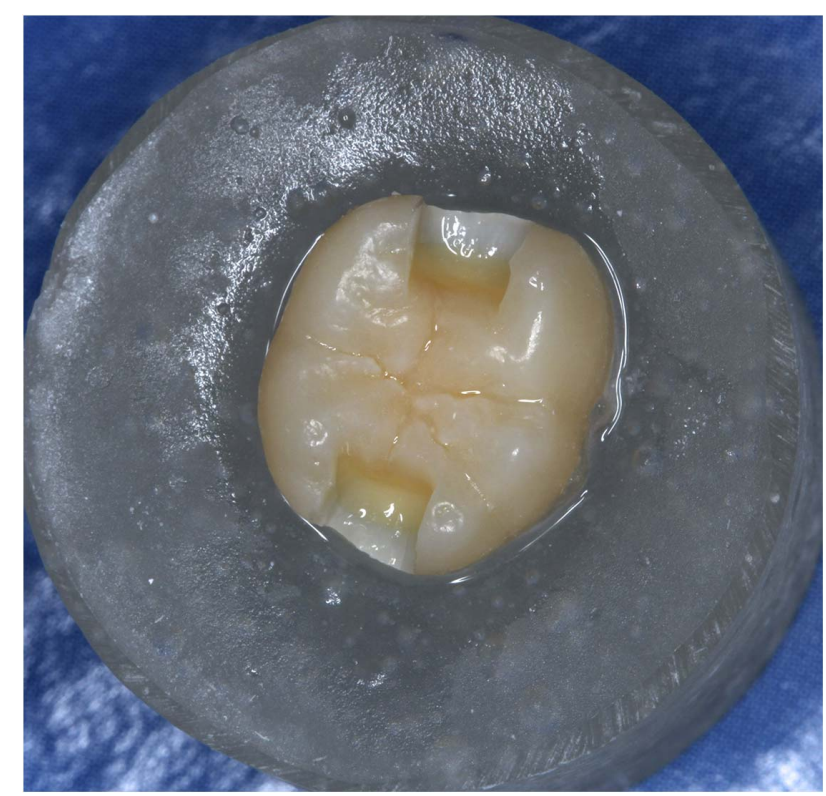

Fig. 1. Mounting the teeth in autopolymerizing acrylic resin and class II box preparation

\section{Cavity preparation}

Standardized Class II box preparations were carried out on the mesial and distal surfaces of each tooth. The dimensions of the preparation were $4 \mathrm{~mm}$ buccolingually and $4 \mathrm{~mm}$ occluso-gingivally with a pulpal depth of $3 \mathrm{~mm}$ [21]. The gingival floor was prepared coronal to the cementoenamel junction (Fig. 1). The teeth were prepared using a \#245 carbide bur (SS White, Lakewood, NJ, USA) in an air/water-cooled high-speed turbine. A new bur was used after completion of five preparations [22].

\section{Grouping}

The prepared teeth were randomized using a randomization website (www.random.org) into four groups $(n=12)$. All the teeth were etched with $37 \%$ phosphoric acid for $20 \mathrm{~s}$ on enamel and $15 \mathrm{~s}$ on dentin, rinsed with water for $10 \mathrm{~s}$ [23], blot dried with cotton pellet to achieve a moist dentin surface and then bonded with Peak ${ }^{\circledR}$ Universal Bond, according to manufacturer's recommendations. A tofflemire matrix was placed to prevent curing light transmission proximally then restored with different materials to be tested. Materials used in this study are shown in Table $I$.

Group 1: teeth were restored with Filtek ${ }^{\mathrm{TM}}$ Supreme Ultra Universal Restorative incrementally, $2 \mathrm{~mm}$ per increment, placed and cured for $20 \mathrm{~s} /$ increment with an LED curing light (intensity of $595 \mathrm{~mW} / \mathrm{cm}^{3}$ ) [14], the light curing was done at $90^{\circ}$ occlusally in relation to the tooth, $5 \mathrm{~mm}$ away from the tooth with continuous light curing, followed by finishing and polishing. Group 2: teeth were restored in $4-\mathrm{mm}$ bulk with Filtek ${ }^{\mathrm{TM}}$ Supreme Ultra 
Table I Materials used in the study

\begin{tabular}{ll} 
Product, manufacturer & \multicolumn{1}{c}{ Description } \\
\hline Filtek $^{\mathrm{TM}}$ Supreme Ultra & $\begin{array}{l}\text { Conventional nanohybrid resin-based dental } \\
\text { Cnniversal, } 3 \mathrm{M}^{\mathrm{TM}} \text { ESPE }^{\mathrm{TM}}\end{array}$ \\
\hline
\end{tabular}

composite
Filtek $^{\mathrm{TM}}$ Bulk Fill

Flowable Restorative, $3 \mathrm{M}^{\mathrm{TM}} \mathrm{ESPE}^{\mathrm{TM}}$

SureFil ${ }^{\circledR} \mathrm{SDR}^{\circledR}$ flow

Posterior Bulk Fill

Flowable, DENTSPLY

Caulk

Peak ${ }^{\circledR}$ Universal Bond, Ultradent Products, Inc.
A single-component, light curing adhesive that can be used in self-etch or total-etch procedures

One-component, fluoride-containing, visible light cured, radiopaque resin composite restorative material has handling characteristics typical of a "flowable" composite

A single syringe-delivered light-cured adhesive resin with chlorhexidine $(0.2 \%)$
Composition

Bisphenol-A diglycidyl ether dimethacrylate, urethane dimethacrylate (UDMA), triethyleneglycol dimethacrylate (TEGDMA), and bisphenol A polyethylene glycol diether dimethacrylate $(6)$ resins. The filler is a combination of silica filler and zirconia filler

UDMA, bisEMA (6), and Procrylat resins. The fillers are a combination of zirconia/silica

Modified UDMA, ethoxylated ethoxylated Bisphenol A dimethacrylate, and TEGDMA, resins. The filler is a combination of Bariumalumino-fluoro-borosilicate glass and strontium alumino-fluoro-silicate glass

Ethyl alcohol, 2-hydroxyethyl methacrylate
Universal Restorative and cured for $20 \mathrm{~s}$ with an LED curing light (intensity of $595 \mathrm{~mW} / \mathrm{cm}^{3}$ ) [14], the light curing was done at $90^{\circ}$ occlusally in relation to the tooth, $5 \mathrm{~mm}$ away from the tooth with continuous light curing, followed by finishing and polishing. Group 3: teeth were restored in 4-mm bulk with Filtek ${ }^{\mathrm{TM}}$ Bulk Fill Flowable Restorative and cured for $20 \mathrm{~s}$ with an LED curing light (intensity of $595 \mathrm{~mW} / \mathrm{cm}^{3}$ ) [14], the light curing was done at $90^{\circ}$ occlusally in relation to the tooth, $5 \mathrm{~mm}$ away from the tooth with continuous light curing, followed by finishing and polishing. Group 4: teeth were restored in 4-mm bulk with SureFil ${ }^{\circledR}$ SDR $^{\circledR}$ flow and cured for $20 \mathrm{~s}$ with an LED curing light (intensity of $595 \mathrm{~mW} / \mathrm{cm}^{3}$ ) [14], the light curing was done at $90^{\circ}$ occlusally in relation to the tooth, $5 \mathrm{~mm}$ away from the tooth with continuous light curing, followed by finishing and polishing.

The intensity of the light-curing machine was checked after every five curing using radiometer (Demetron, Kerr Corporation, CA, USA).

All the teeth were thermocycled for 2,000 thermal cycles between water baths at $5{ }^{\circ} \mathrm{C}$ and $55^{\circ} \mathrm{C}$ with a 30 -s dwell time, according to previous studies [24-26]. Then, the teeth were coated with two layers of nail polish
(Vinyl shine nail polish; Rimmel London, London, UK), except for a $2.0-\mathrm{mm}$ rim around the restoration, to allow contact of the leakage-tracing agent with the margins of the restoration. Next, the teeth were immersed into a solution of $50 \mathrm{wt} \%$ ammoniacal silver nitrate (Fisher Scientific, USA) $(\mathrm{pH}=9.5)$ for $24 \mathrm{~h}$, followed by $8 \mathrm{~h}$ in photo-developing solution (Eastman Kodak Co, Rochester, NY, USA) [27]. Then, specimens were washed under running water for $1 \mathrm{~min}$. The nail polish was carefully removed with \#15 scalpel (Bard-Parker, USA). Each tooth was embedded in clear epoxy resin material, EpoKwick Resin (Buehler, USA), before it was mesiodistally sectioned with a single section through the center of the restoration, and then measured from the occlusal and gingival surfaces for both restorations placed mesially and distally. Four readings per tooth were recorded.

The specimens were analyzed with a stereomicroscope at $20 \times$ magnification and scored according to the percentage of the dye penetration along the dentin walls, based on the following formula: microleakage percentage $=$ dye penetration from the outer margin $/$ total surface of the restoration. A sample of a stereomicroscope image showing microleakage is displayed in Fig. 2.

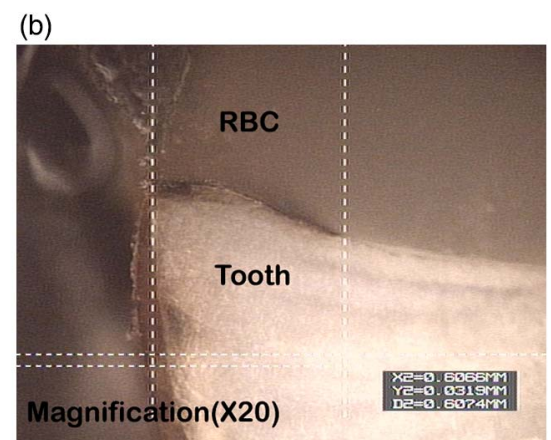

(b) (a)

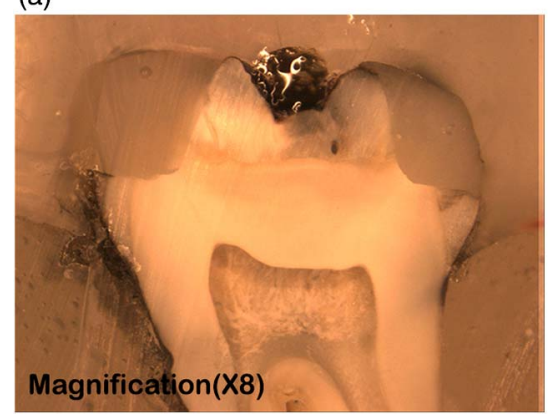

Fig. 2. Sample of microleakage in stereomicroscope imaging (a) Sample of a microleakage $(\times 8)$, (b) Sample of a microleakage $(\times 20)$ 


\section{Statistical analysis}

A statistical software (SAS version 9.2 and SPSS version 19, IL., USA) was used in the analysis. Descriptive statistics were reported in the form of counts and percentages. One-way analysis of variance (ANOVA) was used to compare the four groups in terms of microleakage percentage and dye penetration. The ANOVA test was performed after results of the Kolmogorov-Smirnov test showed normally distributed data. To account for multiple comparisons between groups, the Bonferroni correction was used. The Mann-Whitney-Wilcoxon test was used to compare occlusal and gingival microleakage readings.

\section{Results}

\section{Assessment of dye penetration and microleakage}

Dye penetration and consequently microleakage varied between the groups. One-way ANOVA revealed significance difference between the groups in terms of microleakage $(P<0.01)$ and dye penetration $(P=0.006)$. Both Filtek $^{\mathrm{TM}}$ Bulk Fill Flowable and conventional nanohybrid RBCs showed the least microleakage with a mean (standard deviation) of 1.90 (4.59) and 1.99 (2.24), respectively. SureFil ${ }^{\circledR} \mathrm{SDR}^{\circledR}$ flow flowable composite had a mean (standard deviation) microleakage value of 3.91 (6.63). Finally, the nanohybrid composite in 4-mm bulk showed the highest microleakage, with a mean value of $8.02 \pm 4.01$. The mean and standard deviation values are shown in Table II and Fig. 2.

Given the multiple comparisons, a Bonferroni post hoc test was applied; it revealed that the difference between incremental Filtek ${ }^{\mathrm{TM}}$ Supreme Ultra Universal Restorative and Filtek ${ }^{\mathrm{TM}}$ Bulk Fill Flowable Restorative with respect to dye penetration/microleakage was not statistically significant $(P>0.05)$. However, there was a statistically significant difference between the previous two groups compared with Filtek ${ }^{\mathrm{TM}}$ Supreme Ultra Universal Restorative restored in bulk, in terms of both dye penetration $(P<0.05$ for both) and microleakage $(P<0.05$ and $P<0.05$ for groups 1 and 3 , respectively). On the other hand, the difference between SureFil ${ }^{\circledR}$ SDR $^{\circledR}$ flow and all the other groups was not statistically significant in terms of either dye penetration $(P>0.05$ for groups $1-3)$ or microleakage
$(P=1.000,0.215$, and 1.000 for groups 1,2 , and 3 , respectively). Results are shown in Table III.

The dye penetration/microleakage surfaces were compared using the Mann-Whitney-Wilcoxon test. They showed significantly more gingival microleakage than occlusal microleakage $(P<0.05)$. Gingival microleakage was found in $45 \%$ of the restorations, whereas occlusal microleakage in $18.75 \%$ of the restorations.

\section{Discussion}

In the present in vitro study, we compared the marginal microleakage (silver nitrate uptake) observed with nanohybrid RBCs in two different clinical protocols and two bulk-fill flowable composites. The first protocol used the conventional recommended incremental technique served as a control group and a 4-mm bulk of nanohybrid RBC to be comparable with the tested bulk-fill flowable RBCs, bulk-fill flowable RBC, Filtek ${ }^{\mathrm{TM}}$ Bulk Fill Flowable Restorative $\left(3 \mathrm{M}^{\mathrm{TM}}, \mathrm{ESPE}^{\mathrm{TM}}\right)$, and SureFil ${ }^{\circledR} \mathrm{SDR}^{\circledR}$ flow (DENTSPLY Caulk), according to their manufacturers' recommendations. To simulate clinical conditions, extracted intact human teeth were used in this investigation. After randomization and preparation of the samples, the teeth were kept in distilled water throughout the investigation to prevent dehydration. All cavities were prepared and restored by the same experienced operator. All the samples were thermocycled for 2,000 cycles to resemble aging of the restoration in the oral environment and then were embedded in silver nitrate to detect microleakage.

Based on our results, the first null hypothesis was rejected: the nanohybrid $\mathrm{RBC}$ did not exhibit less marginal leakage than the two bulk-fill flowable RBCs using the incremental technique and exhibited more microleakage using the bulk-filling technique. The second null hypothesis was also rejected: the nanohybrid $\mathrm{RBC}$ when restored in $2-\mathrm{mm}$ increments exhibited less marginal leakage than the $4-\mathrm{mm}$ bulk. The results of this in vitro study revealed that the Filtek ${ }^{\mathrm{TM}}$ Bulk Fill Flowable RBC had the lowest mean value for marginal microleakage. However, the standard deviation was wide compared with the conventional nanohybrid RBC incremental technique, which had a mean value very similar to that of the Filtek ${ }^{\mathrm{TM}}$ Bulk Fill Flowable RBC with a smaller standard deviation. There was no

Table II Total dye penetration $(\mu \mathrm{m})$ and microleakage results (\%) of the four study groups

\begin{tabular}{|c|c|c|c|c|c|}
\hline Mean (standard deviation) & $\begin{array}{c}\text { Filtek Supreme Ultra } \\
\text { Universal, } 3 \mathrm{M}^{\mathrm{TM}} \\
\text { incrementally }\end{array}$ & $\begin{array}{l}\text { Filtek Supreme } \\
\text { Ultra Universal, } \\
3 \mathrm{M}^{\mathrm{TM}} \text { in bulk }\end{array}$ & $\begin{array}{c}\text { Filtek }^{\mathrm{TM}} \text { Bulk Fill } \\
\text { Flowable } \\
\text { Restorative }\end{array}$ & $\begin{array}{c}\text { SureFil }^{\circledR} \text { SDR }^{\circledR} \\
\text { flow }\end{array}$ & $\begin{array}{c}P \\
\text { value }\end{array}$ \\
\hline Total dye penetration $(\mu \mathrm{m})$ & $145.95(162.10)$ & $573.85(284.81)$ & $145.51(357.76)$ & $253.3(424.08)$ & 0.006 \\
\hline Microleakage (\%) & $1.99(2.24)$ & $8.02(4.00)$ & $1.90(4.59)$ & $3.96(6.63)$ & 0.007 \\
\hline
\end{tabular}


AlSagob et al.

Table III Total dye penetration $(\mu \mathrm{m})$ and microleakage (\%) comparison between the four study groups

\begin{tabular}{|c|c|c|c|c|c|}
\hline Variable & Group & Group & Mean difference & Standard error & $P$ value \\
\hline \multirow[t]{12}{*}{ Total dye penetration $(\mu \mathrm{m})$} & \multirow[t]{3}{*}{1} & 2 & -427.90 & 131.34 & 0.013 \\
\hline & & 3 & 0.44 & 131.34 & 1.000 \\
\hline & & 4 & -107.35 & 131.34 & 1.000 \\
\hline & \multirow[t]{3}{*}{2} & 1 & 427.9 & 131.34 & 0.013 \\
\hline & & 3 & 428.34 & 131.34 & 0.013 \\
\hline & & 4 & 320.55 & 131.34 & 0.114 \\
\hline & \multirow[t]{3}{*}{3} & 1 & -0.44 & 131.34 & 1.000 \\
\hline & & 2 & -428.34 & 131.34 & 0.013 \\
\hline & & 4 & -107.79 & 131.34 & 1.000 \\
\hline & \multirow[t]{3}{*}{4} & 1 & 107.35 & 131.34 & 1.000 \\
\hline & & 2 & -320.55 & 131.34 & 0.114 \\
\hline & & 3 & 107.79 & 131.34 & 1.000 \\
\hline \multirow[t]{12}{*}{ Microleakage (\%) } & \multirow[t]{3}{*}{1} & 2 & -6.03 & 1.89 & 0.016 \\
\hline & & 3 & 0.09 & 1.89 & 1.000 \\
\hline & & 4 & -1.93 & 1.89 & 1.000 \\
\hline & \multirow[t]{3}{*}{2} & 1 & 6.03 & 1.89 & 0.016 \\
\hline & & 3 & 6.11 & 1.89 & 0.014 \\
\hline & & 4 & 4.10 & 1.89 & 0.215 \\
\hline & \multirow[t]{3}{*}{3} & 1 & -0.09 & 1.89 & 1.000 \\
\hline & & 2 & -6.11 & 1.89 & 0.014 \\
\hline & & 4 & -2.012 & 1.89 & 1.000 \\
\hline & \multirow[t]{3}{*}{4} & 1 & 1.93 & 1.89 & 1.000 \\
\hline & & 2 & -4.10 & 1.89 & 0.215 \\
\hline & & 3 & 2.01 & 1.89 & 1.000 \\
\hline
\end{tabular}

Group 1: Filtek ${ }^{\mathrm{TM}}$ Supreme Ultra Universal Restorative incrementally; Group 2: Filtek ${ }^{\mathrm{TM}}$ Supreme Ultra Universal Restorative in bulk; Group 3: Filtek $^{\mathrm{TM}}$ Bulk Fill Flowable Restorative; Group 4: SureFil ${ }^{\circledR} \mathrm{SDR}^{\circledR}$ flow

statistically significant difference in microleakage between the two groups, which is a promising result in regard to the bulk-fill flowable composite. However, it is still more predictable to use the incremental technique. These findings are in accordance with those reported by Czasch and Ilie [14] and Moorthy et al. [15]. Of note in the latter study, the cavity design had fairly shallow areas, which might not reflect the bulkfilling properties of the material.

Although the SureFil ${ }^{\circledR}$ SDR $^{\circledR}$ flow showed higher microleakage than that is seen with the Filteck ${ }^{\mathrm{TM}}$ Bulk Fill Flowable RBC and the nanohybrid RBC incremental technique, the difference was not statistically significant. The difference was also not statistically significant when the nanohybrid RBC was placed in $4-\mathrm{mm}$ bulk. These results were in agreement with those of Roggendorf et al. [13], who found that $4 \mathrm{~mm}$ of SDR ${ }^{\circledR}$ had no detrimental effect in comparison with the conventional composite in terms of microleakage.
Conventional nanohybrid RBC when placed in 4-mm bulk showed the highest microleakage, as anticipated, significantly different from the Filtek ${ }^{\mathrm{TM}}$ Bulk Fill Flowable $\mathrm{RBC}$ and the nanohybrid RBC incremental technique. This is primarily due to the limitations of the physical properties of the material; the incremental technique is the gold standard for conventional RBC to achieve good physical properties. This result is in agreement with numerous studies [28-36], which reported that the inadequate resin polymerization adversely affects the RBC physical properties, reduces the bond strength to the tooth, and increases marginal wear and breakdown. It is noteworthy that the recommended 20-s curing time is shade-dependent. That time is applicable only for the universal shade that was used in this study for both materials and the results showed that it was sufficient. For the other shades of Filtek ${ }^{\mathrm{TM}}$ Bulk Fill Flowable, the manufacturer's recommendation was $40 \mathrm{~s}$. The results of this study are in agreement with those of Czasch and 
Ilie [14], who stated that the polymerization time of $20 \mathrm{~s}$ for $4 \mathrm{~mm}$ is appropriate.

Nevertheless, the results showed that the simplified application procedure with 4-mm bulk of bulk-fill flowable RBCs and $20 \mathrm{~s}$ of curing, particularly for Filtek ${ }^{\mathrm{TM}}$ Bulk Fill Flowable, did not negatively affect the polymerization shrinkage. Consequently, the marginal microleakage is of great appeal. The results of this study also revealed that the marginal microleakage in the gingival surface was statistically higher than that in the occlusal surface. This result contradicts the study conducted by Deliperi and Bardwell [3], as they found no significant difference in dye penetration between the occlusal and gingival microleakage scores for most of the comparison groups, except one that yielded more dye penetration at the occlusal surface than at the gingival. This could be due to their study design; they were examining different adhesive techniques for the conventional restorative $\mathrm{RBC}$ protocol in $2-\mathrm{mm}$ increments. In contrast, this study examined a bulk-filling protocol. The deepest area of the composite bulk may not have been adequately polymerized.

Interestingly, the difference in microleakage could not be predicted between Filtek ${ }^{\mathrm{TM}}$ Bulk Fill Flowable and SDR $^{\circledR}$, since both are from the same material type, have similar properties, and have enhanced translucency [37] that promotes light transmittance and enables adequate curing efficiency up to 4-mm bulk of composite. However, the average particle size of Filtek ${ }^{\mathrm{TM}}$ is $0.6 \mathrm{~nm}$, whereas the $\mathrm{SDR}^{\circledR}$ average particle size is $4.2 \mathrm{~nm}$. This difference in particle size may have contributed to hindering light penetration of the light cure and consequently polymerization. Another explanation could be the novel monomer composition [3].

\section{Study limitations and further research}

The methodology used for bulk-fill flowable RBC was application of the material in 4-mm bulk to fill the entire cavity prepared. This methodology is in contrast to that used in the clinical setting, where the manufacturer recommends use of the $4-\mathrm{mm}$ bulk as the base layer for boxes deeper than $4 \mathrm{~mm}$, followed by a capping layer (open sandwich technique). This is primarily due to the difficulty in handling the flowable consistency for replicating the anatomy of the tooth, not due to material limitation. For this reason, the study design was adequate for the intended polymerization and microleakage testing of the material.

In this in vitro study, the polymerization method was specified, using $90^{\circ}$ occlusally in relation to the tooth, $5 \mathrm{~mm}$ away from the tooth with continuous light curing. Use of tofflemire matrix to prevent curing light transmission proximally was necessary for the purpose of this study (i.e., evaluating the microleakage of 4 - $\mathrm{mm}$ bulk fill of $\mathrm{RBC}$ ). The $5-\mathrm{mm}$ distance was selected to simulate the clinical setting. However, curing $90^{\circ}$ from the occlusal surface is difficult to achieve in some of the situations where the restoration is hard to reach, and it is not the typical clinical curing method. But it was used in this study to standardize the restoration and curing method procedure for all the samples. Perhaps future studies could involve different cavity designs that enable a more relevant clinical protocol, using a clear matrix and different curing directions.

In this study, we adopted a commonly used aging method to simulate the degradation of bond over time in the oral cavity, aging by thermocycling. The efficacy of thermocycling in simulating clinical aging has been the subject of controversy among researchers [38, 39]. Although it is the most frequently used method of aging for microleakage evaluation up to now, there is no consensus in the literature for a relevant regimen for aging [19]. Other aging methods include water storage and thermomechanical loading. Previous studies showed statistically significant difference between thermocycling and water storage, thermocycling being superior [40]. Furthermore, thermomechanical loading is superior to thermocycling, with a statistically significant difference [41]. Thermomechanical loading is recommended for use in future studies, as it is more analogous to the oral condition.

Due to time and resources limitation, the evaluation of dye penetration was done in a two-dimensional view, using a single section through the center of the restoration. This might have resulted in an underestimate of the results. Future studies may employ multiple sectioning of the teeth to obtain a three-dimensional view, thus presenting more accurate results. In addition, the evaluation of dye penetration was done using a stereomicroscope at $20 \times$ magnification, which was sufficient to evaluate the dye penetration. However, it would be interesting to examine the mode of failure using a scanning electron microscope in future studies.

\section{Conclusions}

Within the limitations of the study, the following conclusions can be drawn:

1. Among the compared groups, Filtek ${ }^{\mathrm{TM}}$ Bulk Fill Flowable Restorative and the conventional nanohybrid composite exhibited the least microleakage. However, the conventional nanohybrid composite is still more predictable to use.

2. The polymerization time of $20 \mathrm{~s}$ for the universal shade of both bulk-fill flowable composites seems appropriate. 
3. As anticipated, with the bulk-filling technique, the marginal microleakage in the gingival surface was higher than that in the occlusal surface.

\section{*** \\ Funding sources: No financial support was received for this study.}

Conflict of interest: The authors declare no conflict of interest.

Authors' contribution: EIA: manage of research, literature search, lab procedures, data analysis, and manuscript preparation; DNB and AOA: research advisor; SGK and PCS: research committee.

Acknowledgements: This manuscript was adapted from thesis submitted to School of Dental Medicine, Tufts University, Boston, USA in partial fulfillment of the requirement for the degree of Master of Science.

\section{References}

1. Brunthaler A, Konig F, Lucas T, Sperr W, Schedle A: Longevity of direct resin composite restorations in posterior teeth. Clin Oral Investig 7, 63-70 (2003)

2. Sarrett DC: Clinical challenges and the relevance of materials testing for posterior composite restorations. Dent Mater 21, 9$20(2005)$

3. Deliperi S, Bardwell DN: An alternative method to reduce polymerization shrinkage in direct posterior composite restorations. J Am Dent Assoc 133, 1387-1398 (2002)

4. Unterbrink GL, Liebenberg WH: Flowable resin composites as "filled adhesives": Literature review and clinical recommendations. Quintessence Int 30, 249-257 (1999)

5. Rullmann I, Schattenberg A, Marx M, Willershausen B, Ernst CP: Photoelastic determination of polymerization shrinkage stress in low-shrinkage resin composites. Schweiz Monatsschr Zahnmed 122, 294-299 (2012)

6. Park J, Chang J, Ferracane J, Lee IB: How should composite be layered to reduce shrinkage stress: Incremental or bulk filling? Dent Mater 24, 1501-1505 (2008)

7. Kim ME, Park SH: Comparison of premolar cuspal deflection in bulk or in incremental composite restoration methods. Oper Dent 36, 326-334 (2011)

8. Davidson CL: Resisting the curing contraction with adhesive composites. J Prosthet Dent 55, 446-447 (1986)

9. Lutz F, Krejci I, Barbakow F: Quality and durability of marginal adaptation in bonded composite restorations. Dent Mater 7, 107$113(1991)$

10. Ikeda I, Otsuki M, Sadr A, Nomura T, Kishikawa R, Tagami J: Effect of filler content of flowable composites on resin-cavity interface. Dent Mater J 28, 679-685 (2009)

11. Burgess J, Cakir D: Comparative properties of low-shrinkage composite resins. Compend Contin Educ Dent 31, 10-15 (2010)

12. Fleming GJP, Awan M, Cooper PR, Sloan AJ: The potential of a resin-composite to be cured to a $4 \mathrm{~mm}$ depth. Dent Mater 24,522 $529(2008)$

13. Roggendorf MJ, Kramer N, Appelt A, Naumann M, Frankenberger $\mathrm{R}$ : Marginal quality of flowable $4-\mathrm{mm}$ base vs. conventionally layered resin composite. J Dent 39, 643-647 (2011)

14. Czasch P, Ilie N: In vitro comparison of mechanical properties and degree of cure of bulk fill composites. Clin Oral Investig 17, 227235 (2013)

15. Moorthy A, Hogg CH, Dowling AH, Grufferty BF, Benetti AR, Fleming GJ: Cuspal deflection and microleakage in premolar teeth restored with bulk-fill flowable resin-based composite base materials. J Dent 40, 500-505 (2012)

16. Van Ende A, De Munck J, Van Landuyt KL, Poitevin A, Peumans M, Van Meerbeek B: Bulk-filling of high C-factor posterior cavities: Effect on adhesion to cavity-bottom dentin. Dent Mater 29, 269277 (2013)

17. Rengo C, Goracci C, Juloski J, Chieffi N, Giovannetti A, Vichi A, Ferrari M: Influence of phosphoric acid etching on microleakage of a self-etch adhesive and a self-adhering composite. Aust Dent J 57, 220-226 (2012)

18. Guzman-Ruiz S, Armstrong SR, Cobb DS, Vargas MA: Association between microtensile bond strength and leakage in the indirect resin composite/dentin adhesively bonded joint. J Dent $29,145-$ $153(2001)$

19. Raskin A, D'Hoore W, Gonthier S, Degrange M, Dejou J: Reliability of in vitro microleakage tests: A literature review. J Adhes Dent 3, 295-308 (2001)

20. de Almeida JB, Platt JA, Oshida Y, Moore BK, Cochran MA, Eckert GJ: Three different methods to evaluate microleakage of packable composites in class II restorations. Oper Dent 28, 453$460(2003)$

21. Bogra P, Gupta S, Kumar S: Comparative evaluation of microleakage in class II cavities restored with Ceram X and Filtek P-90: An in vitro study. Contemp Clin Dent 3, 9-14 (2012)

22. Cenci M, Demarco F, de Carvalho R: Class II composite resin restorations with two polymerization techniques: Relationship between microtensile bond strength and marginal leakage. J Dent 33, 603-610 (2005)

23. Duarte S Jr, de Freitas CR, Saad JR, Sadan A: The effect of immediate dentin sealing on the marginal adaptation and bond strengths of total-etch and self-etch adhesives. J Prosthet Dent 102, 1-9 (2009)

24. Medina AD, de Paula AB, de Fucio SB, Puppin-Rontani RM, Correr-Sobrinho L, Sinhoreti MA: Marginal adaptation of indirect restorations using different resin coating protocols. Braz Dent J 23, 672-678 (2012)

25. Jafarzadeh Kashi TS, Erfan M, Rakhshan V, Aghabaigi N, Tabatabaei FS: An in vitro assessment of the effects of three surface treatments on repair bond strength of aged composites. Oper Dent 36, 608-617 (2011)

26. Blunck U, Zaslansky P: Enamel margin integrity of class I onebottle all-in-one adhesives-based restorations. J Adhes Dent 13, 23$29(2011)$

27. Hebling J, Pashley DH, Tjaderhane L, Tay FR: Chlorhexidine arrests subclinical degradation of dentin hybrid layers in vivo. J Dent Res 84, 741-746 (2005)

28. Federlin M, Price R: Improving light-curing instruction in dental school. J Dent Educ 77, 764-772 (2013)

29. Calheiros FC, Daronch M, Rueggeberg FA, Braga RR: Degree of conversion and mechanical properties of a BisGMA:TEGDMA composite as a function of the applied radiant exposure. J Biomed Mater Res B Appl Biomater 84, 503-509 (2008)

30. Fan PL, Schumacher RM, Azzolin K, Geary R, Eichmiller FC: Curing-light intensity and depth of cure of resin-based composites tested according to international standards. J Am Dent Assoc 133, 429-434; quiz 491-493 (2002)

31. Shortall A, El-Mahy W, Stewardson D, Addison O, Palin W: Initial fracture resistance and curing temperature rise of ten contemporary resin-based composites with increasing radiant exposure. J Dent 41 , 455-463 (2013)

32. Lohbauer U, Rahiotis C, Kramer N, Petschelt A, Eliades G: The effect of different light-curing units on fatigue behavior and degree of conversion of a resin composite. Dent Mater 21, 608-615 (2005)

33. Ferracane JL, Mitchem JC, Condon JR, Todd R: Wear and marginal breakdown of composites with various degrees of cure. J Dent Res 76, 1508-1516 (1997) 
34. Vandewalle KS, Ferracane JL, Hilton TJ, Erickson RL, Sakaguchi RL: Effect of energy density on properties and marginal integrity of posterior resin composite restorations. Dent Mater 20, 96-106 (2004)

35. Zhu S, Platt J: Curing efficiency of three different curing modes at different distances for four composites. Oper Dent 36, 362-371 (2011)

36. Xu X, Sandras DA, Burgess JO: Shear bond strength with increasing light-guide distance from dentin. J Esthet Restor Dent 18, 19-27; discussion 28 (2006)

37. Lassila LV, Nagas E, Vallittu PK, Garoushi S: Translucency of flowable bulk-filling composites of various thicknesses. Chin J Dent Res 15, 31-35 (2012)
38. Doerr CL, Hilton TJ, Hermesch CB: Effect of thermocycling on the microleakage of conventional and resin-modified glass ionomers. Am J Dent 9, 19-21 (1996)

39. Yap AU: Effects of storage, thermal and load cycling on a new reinforced glass-ionomer cement. J Oral Rehabil 25, 40-44 (1998)

40. Al-Maqtari AA, Lui JL: Effect of aging on coronal microleakage in access cavities through metal ceramic crowns restored with resin composites. J Prosthodont 19, 347-356 (2010)

41. Koyuturk AE, Kusgoz A, Ulker M, Yesilyurt C: Effects of mechanical and thermal aging on microleakage of different fissure sealants. Dent Mater J 27, 795-801 (2008) 